\title{
Vehicle-carried Remote Control System Based on ARM
}

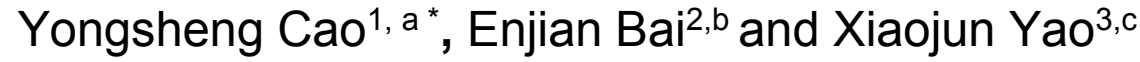 \\ ${ }^{1}$ Information Science and Technology College, Donghua University, Songjiang, Shanghai, China \\ ${ }^{2}$ Information Science and Technology College, Donghua University, Songjiang, Shanghai, China \\ IInformation Science and Technology College, Donghua University, Songjiang, Shanghai, China \\ a110900420@mail.dhu.edu.cn, bbaiej@dhu.edu.cn, c377188753@qq.com
}

\begin{abstract}
Keywords: Smart life; Vehicle-carried system; Remote control system; 4G; Embedded Intelligent Platform

Abstract. Aiming at the lack of intelligentialize of low-end vehicle, the wireless remote control system based on ARM vehicle-carried control system is designed by combining the ARM control and 4G wireless transmission. The integrated structure of the system and data communication and signal control between modules are introduced. In order to strengthen the owner mastery of the vehicle, the system possess the expandable function of $2 \mathrm{D}$ modeling using laser ranging module. The experiment of remote control indicates that this strategy can be used in the rapid intelligence of low-end intelligent car.
\end{abstract}

\section{Introduction}

With the development of economy, the car has become a necessity in our life. However, some high-grade cars' intelligent level is very high, but the price is very expensive. And the demand of intelligence can only be passively accepted rather than be actively configured. Therefore, it ' $s$ significant to developed a movable and customized wireless control system based on the embedded vehicle control system.

$4 \mathrm{G}$ network has $100 \mathrm{Mbps}$ download speed and $20 \mathrm{Mbps}$ upload speed, and the communication service is cheaper than ever before. On-board system can send the warning information by $4 \mathrm{G}$ module.

Combining the high speed wireless data transmission of $4 \mathrm{G}$ network and the powerful control function of ARM, we can make the wireless remote monitoring of the vehicle through sending text messages.

Furthermore, with more and more vehicles entering our life, more and more novice pilots emerge. The cause of many traffic accidents lies in the drivers' lack of control of car body. Combined with the laser ranging sensors, we made the plane modeling of the car body, in order to strengthen the control of car body, which will reduce vehicle impact accident.

\section{The Whole Structure Of System}

The whole structure of system is shown in Figure 1. The system has two components: one part is $4 \mathrm{G}$ module and its peripheral circuit, which sends text messages and embedded device[1] which undertake communications between ARM and $4 \mathrm{G}$ module and vibration sensor, display screen and mobile phone. The function of display screen is to display the operation interface. The other is laser range sensor and embedded device which is used for some process related with laser ranging sensor and graphic modeling. 


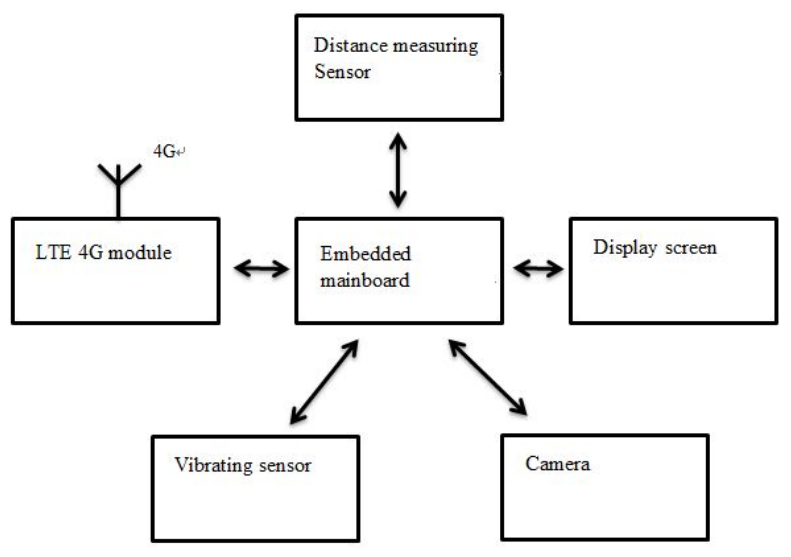

Fig. 1. The structure of the system

4G module selects the Huawei ME906E module in the system. This module supports Mobile 3G, $4 \mathrm{G}$ and Unicom 3G, 4G, providing short message service for the system. ARM embedded system [2] selects Samsung S3C6410 CPU, ARM1176JZF-S kernel, which is connected with 4G module by USB2.0.

When vibration sensor reached the threshold, the camera would be opened to record video for a period of time, the video is stored in the memory for 5 times. When recording the video for the 6 times, the memory card will record circularly. Therefore, we need backup the video file [3] in time, but in the short time we need not worry too much. When the camera is opened, the warning message will be sent to the owner of the car to remind the owner of the potential risk. When owners need to control the car body accurately, they can use laser ranging sensors to make graphic modeling in a certain range. The display screen will show the owner the distance of the car and the obstruction.

In this way, we can avoid traffic accidents effectively and we have a good control of the distance, which can improve the comfort by strengthening the control of the car.

\section{The Communication Of The System}

A.Data interaction between vibrating sensor and ARM

In order to avoid the vibration sensor [4] being triggered by mistake, the system used the voltage comparator LM393 to pre-process the binary signals produced by vibrations. Then the system filter interference signals [5] and A/D converter integrated in the ARM samples the signal for the purpose of converting analog signal to digital signal. The A/D converter transforms the analog signals which are easy to identify into digital signals. The basic task of the converter is to transform the input of continuous range amplitude into a set of discrete digital code subsets. This includes quantization and coding. We select the internal reference voltage and ADC will choose its internal band-gap reference voltage circuit. The conversion range of $\mathrm{ADC}$ is $0 \sim 3.3 \mathrm{~V}$, the computation formula of the result of converting is as follows.

$$
\text { result }=\left\{\begin{array}{c}
0 \quad \text { input } \leq 0 \mathrm{~V} \\
4096 \times \frac{\text { input }- \text { zero_po int }}{V_{\text {ref }}} \quad 0 \mathrm{~V}<\text { input }<V_{\text {ref }} \\
4096 \text { input } \geq 3.3 \mathrm{~V}
\end{array}\right.
$$

Afterwards, we process the discrete signal by using the algorithm, when the digital value reached threshold value, the ARM device will open the camera to record video. The structure diagram of the system is shown in Figure 2. The circuit of vibration sensor is shown in Figure 3. 


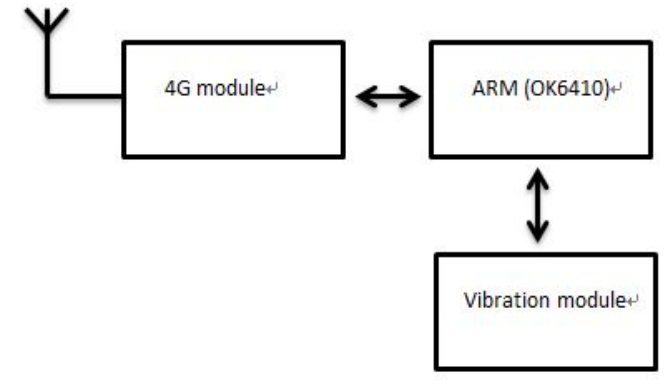

Fig. 2. The structure diagram of the system

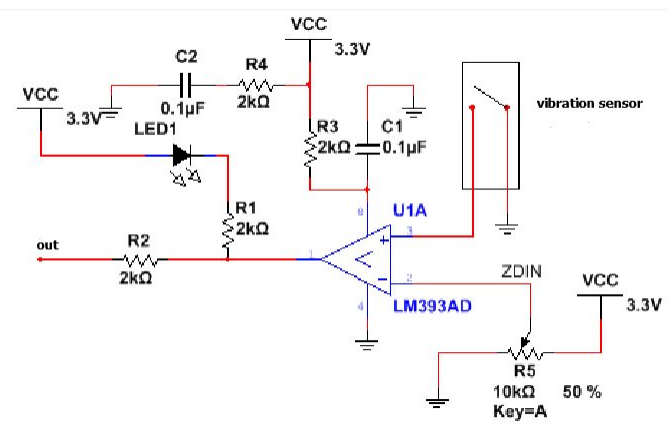

Fig.3. The circuit diagram of vibration sensor

B. The data interaction between ARM and $4 \mathrm{G}$ module

4G module communicates with ARM with the method of serial communication, using AT order to make information exchange and transmission. The communication mode of ARM and $4 \mathrm{G}$ is as follows.

ARM sends AT command to the $4 \mathrm{G}$ module. If the return result is $\mathrm{OK}^{\prime}$, it proves that $4 \mathrm{G}$ module is well-connected with ARM. Then ARM will send AT+CPIN command to 4G module. If the returned result is 'READY', then it means SIM works and it doesn' t need password. Common AT command is as shown in Figure 4.

\begin{tabular}{|l|l|}
\hline Command & Description \\
\hline AT + CMGD & DELETE SMS MESSAGE \\
\hline AT+CMGF & SELECT SMS MESSAGE FORMAT \\
\hline AT + CMGL & LIST SMS MESSAGES FROM PREFERRED STORE \\
\hline AT + CMGR & READ SMS MESSAGE \\
\hline AT CMGS & SEND SMS MESSAGEF \\
\hline AT + CMGW & WRITE SMS MESSAGE TO MEMORY \\
\hline AT + CMSS & SEND SMS MESSAGE FROM STORAGE \\
\hline AT + CMGC & SEND SMS COMMAND \\
\hline AT + CNMI & NEW SMS MESSAGE INDICATIONS \\
\hline AT + CPMS & PREFERRED SMS MESSAGE STORAGE \\
\hline AT + CRES & RESTORE SMS SETTINGS \\
\hline AT + CSAS & SAVE SMS SETTINGS \\
\hline AT + CSCA & SMS SERVICE CENTER ADDRESS \\
\hline AT + CSCB & SELECT CELL BROADCAST SMS MESSAGES \\
\hline AT + CSDH & SHOW SMS TEXT MODE PARAMETERS \\
\hline AT + CSMP & SET SMS TEXT MODE PARAMETERS \\
\hline AT + CSMS & SELECT MESSAGE SERVICE \\
\hline
\end{tabular}

Fig.4. AT command

C. Data interaction between ARM and laser range sensor

The data output of laser range sensor HJ-40A is UART serial port(TTL,19200bps) or serial port RS232. Forlinx OK6410 main board has 4 serial ports, including a five-line RS-232 serial port(DB9) and three three-line TTL serial ports(20pin 2.0mm spacing connector). ARM reads the data of laser ranging sensor by RS232 and shows the image based on the distance which is convenient to the owner to control the car.

\section{The Design Of Software}

\section{A.Main workflow of the system}

From Figure 1, we know the relationship of each part in the system. All peripheral modules of the system are connected with the relevant interface of OK6410, which will exchange data with ARM. Therefore, ARM should be initialized firstly after powering up, ensuring that each module works.

Firstly, we wrote the program of the vibration sensor module [6], camera [7], laser range sensor and $4 \mathrm{G}$ module. Then, the main program sets registers that have the function of closing some modules selectively achieving the goal of saving energy. In idle mode, CPU kernel stops working and just keeps the I/O controller which can wake up the CPU working normally. When the vibration signal wakes up CPU, a signal will be sent to camera then camera will be open for a period of time and the video will be stored in storage card. At the same time, the system is connected with $4 \mathrm{G}$ network by the $4 \mathrm{G}$ module and sends warning messages to the owner of the car. The complete workflow is shown in Figure 5. 


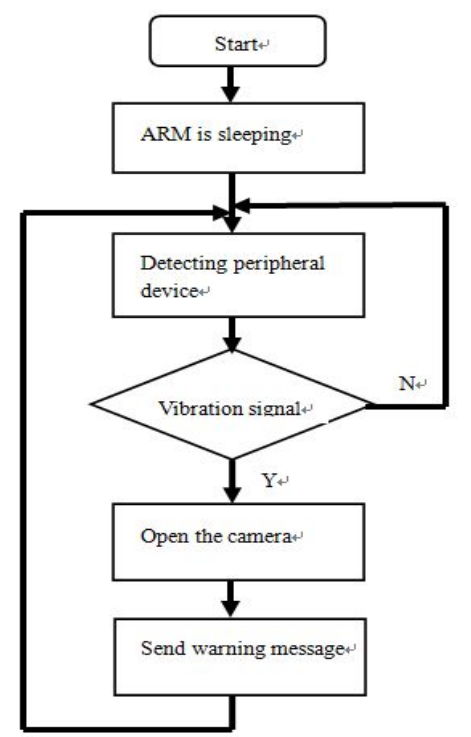

Fig.5. The workflow of the main program

B. The workflow of the camera

This system is based on Linux. There is an algorithm transforming V4L2 to BMP [8] in the program of camera. The code of the algorithm is as follows.

\section{Summary}

This system combines the advantage of broadband wireless data transmission of $4 \mathrm{G}$ network and the strong programmable function of ARM. It possesses cost-effective on-board wireless monitoring function. Meanwhile, the laser range sensor extracts the information of the distance, which can let the owner have a good control of the car body. This shows the practicability, reliability and the convenience of quadratic transformation of the system.

\section{References}

[1] Miao-Tian Wang, $\mathrm{T}$ he design and examples of the embedded system. Beijing: Tsinghua University Press, 2002.

[2] Li-Gong Zhou, Ming-Ji Chen, Yu Chen, ARM embedded and Linux research and transplantation on ARM, Computer knowledge and technology, 2005, 30(4): 3-4.

[3] Li-Hua Song, Ke Gao. Embedded USB camera driver implementation, The Northern Industrial University, Computer Engineering, 2010.5.

[4] Xue-Shan Yang, Engineering vibration measuring instruments and testing technology, Beijing: Metrology publishing house in China, 2001:3-39.

[5] Chen T,Cao X.A New DTMF Detection Scheme Based on NDFT Goertzel Filter [J],Journal of Southwest University : Natural Science Edition,2008,30(1):152-155.

[6] Jing-Jie Zhou, Zhong-Quan Luan, Xiao-Li Xu, Part of hardware design of vibration monitor based on ARM, Journal of Beijing institute of machinery industry, 2006, 21(3): 12 16.

[7] Yong-Qing Wang, The image acquisition of USB camera based on ARM920T under Linux.

[8] Li-Li Wu, The development and loading of embedded USB driver, Beijing: Beijing University of Posts And Telecommunications, Information science and technology, 2009.4. 